\title{
Tratamiento endoluminal de disección aguda complicada en aorta descendente. Comunicación de un caso
}

\author{
Mario Fava $\mathrm{P}^{1}$, Manuel Espíndola ${ }^{2}$, Hernán Bertoni ${ }^{3}$, \\ M Soledad Loyola $Z^{1}$, Luis Meneses $Q^{1}$, Mauricio Maureira². \\ Endoluminal stent-graft placement \\ for acute dissection of the \\ descending aorta complicated \\ with rupture. Report of one case
}

\begin{abstract}
Acute dissection of the aorta, although not common, has early and highly lethal complications. The type A dissection is treated with surgery. Patients with type B dissections are treated with surgery if they have complications like rupture, growth or visceral ischemia. Surgery, however, has complications such as spinal cord ischemia. Endovascular grafts have less mortality and complications. We report a 59 years old male patient with a type B dissection complicated with rupture. He was treated successfully with the placement of an endoluminal graft. He was discharged five days after the procedure in good conditions. After one year of follow up, the patient remains asymptomatic (Rev Méd Chile 2006; 134: 1024-9).
\end{abstract}

(Key words: Aortic aneurysm, thoracic; Aortic rupture; Blood vessel prosthesis)

\begin{abstract}
Recibido el 29 de septiembre, 2005. Aceptado el 20 de enero, 2006.
${ }_{1}^{1}$ Departamento de Radiología Intervencional, Pontificia Universidad Católica de Chile. Santiago de Chile. ${ }^{2}$ Servicio de Cirugía Vascular. Hospital DIPRECA. Santiago de Chile. ${ }^{3}$ Servicio de Radiología Intervencional y Cirugía Cardiotorácica, Hospital Italiano. Buenos Aires. Argentina.
\end{abstract}

$\mathrm{L}$ a disección aórtica clásica consiste en una _separación de la capa íntima y media de la pared arterial, determinada por una rotura intimal que conecta el lumen aórtico con la capa media, creando un lumen falso con persistencia del

Correspondencia a: Dr. Luis Meneses Quiroz. Marcoleta \#367. Departamento de Radiología. Fono: 3543486.

E mail: lmeneseq@puc.cl verdadero. Tradicionalmente, se han clasificado de acuerdo a los segmentos de la aorta que compromete. Una de las más usadas es la clasificación de Standford, que las divide en dos grupos, las tipo A que comprometen la aorta ascendente y las que no lo hacen y que corresponden al tipo $\mathrm{B}^{1}$.

La incidencia de esta patología es estimada en 5-30 pacientes por millón de habitantes por año ${ }^{2}$. Las complicaciones son precoces y altamente letales $^{3,4}$. The International Registry of Acute Aor- 
tic Dissection (IRAD), que consiste en registros de 12 centros de referencia en distintos países, señala una mortalidad global de $26 \%$ en pacientes sometidos a algún tipo de intervención y $58 \%$ en quienes no se realizó ningún procedimiento dado el contexto clínico, como edad avanzada o patologías asociadas. En aquellos con disección tipo B que requirieron intervención por alguna complicación, por ejemplo por rotura, la mortalidad alcanzó a un tercio ${ }^{5}$.

El manejo tradicional en disección aórtica tipo A, es cirugía primaria con interposición de prótesis, lo que exige diagnóstico precoz. Entre las complicaciones descritas en esta cirugía está la isquemia medular, con paraplejia y mortalidad relacionada a múltiples factores, como pérdida sanguínea, insuficiencia renal e insuficiencia respiratoria ${ }^{6-8}$. La técnica quirúrgica con hipotermia y paro circulatorio ha disminuido la morbimortalidad, sin embargo, la necesidad de heparinización aumenta el riesgo de hemorragia fatal, especialmente si existe trauma craneal o pulmonar asociado ${ }^{9,10}$.

En la actualidad, ha aparecido la opción de tratamiento endovascular, que es menos invasiva (en los casos de disección aórtica tipo B), existiendo múltiples trabajos que reportan experiencias exitosas en el tratamiento de patología aguda aórtica ${ }^{11-13}$.

Nosotros comunicamos un caso de disección aguda de aorta torácica descendente, complicada con hemotórax y hemopericardio, tratada exitosamente mediante prótesis endovascular, con seguimiento a mediano plazo sin complicaciones.

\section{CASO CLÍNICO}

Paciente de sexo masculino de 59 años, hipertenso, tabáquico y dislipidémico. Comenzó con dolor torácico retroesternal bajo, opresivo, irradiado a ambos hipocondrios y a espalda, por lo que decidió consultar en servicio de urgencia cinco horas después del inicio de los síntomas.

$\mathrm{Al}$ examen físico estaba vígil, orientado, con presión arterial de 140/90, frecuencia cardiaca de 80 lat/min, con pulsos distales simétricos y normales, sin otros hallazgos relevantes.

Un electrocardiograma practicado al paciente sólo demostró hipertrofia ventricular izquierda. La radiografía no presentó hallazgos relevantes.
Debido a la persistencia del fuerte dolor torácico y para descartar disección aórtica, se solicitó una angiografía por tomografía axial computada de tórax y abdomen (Angio-Tac), que mostró flap de disección de aspecto agudo, que se extendía desde el tercio distal de la aorta descendente hasta el nivel de los hilios renales, con permeabilidad del falso lumen y signos de hemotórax menor y hemopericardio. Se diagnosticó entonces disección aórtica aguda tipo B, complicada por rotura (Figura 1).

Debido a la complicación de la disección, anatomía favorable de la lesión y de los accesos vasculares, se decidió colocación de prótesis endovascular.

Se realizó el procedimiento bajo anestesia general, con acceso a través de arterias femorales e hipotensión controlada, instalándose prótesis endovascular $\left(\right.$ Talent ${ }^{\circledR}$ ) de $34 \mathrm{~mm}$ de diámetro y $130 \mathrm{~mm}$ de longitud en la aorta torácica con resultado técnico adecuado, cobertura de sitio de fractura proximal y sin complicaciones inmediatas (Figura 2).

En estudio de control se comprobó adecuada posición de endoprótesis con exclusión del falso lumen de la aorta torácica, sólo con permeabilidad de éste distal a origen de arteria mesentérica inferior, el que no había aumentado con respecto a examen de ingreso (Figura 3). Los accesos vasculares no presentaban complicaciones. Se decidió el alta cinco días después del procedimiento, con el paciente en buenas condiciones.

En control un año después del procedimiento, el paciente se encontraba en buenas condiciones generales, con Angio-Tac que no demostró complicaciones en la endoprótesis, con exclusión total del falso lumen torácico y sin variaciones con respecto a examen de alta (Figura 4).

\section{DISCUSIÓN}

La disección aórtica aguda tipo A es una emergencia, con mortalidad descrita de $1 \%$ por hora ${ }^{15}$, de allí la importancia del diagnóstico precoz, en que la Angio-Tac es una opción fundamental ${ }^{6,16-17}$.

La reparación quirúrgica de las disecciones tipo $B$ se ha reservado cuando existe expansión aguda del falso lumen torácico $(>4,5 \mathrm{~cm})$, rotura (contenida o no), dolor persistente a pesar de un 

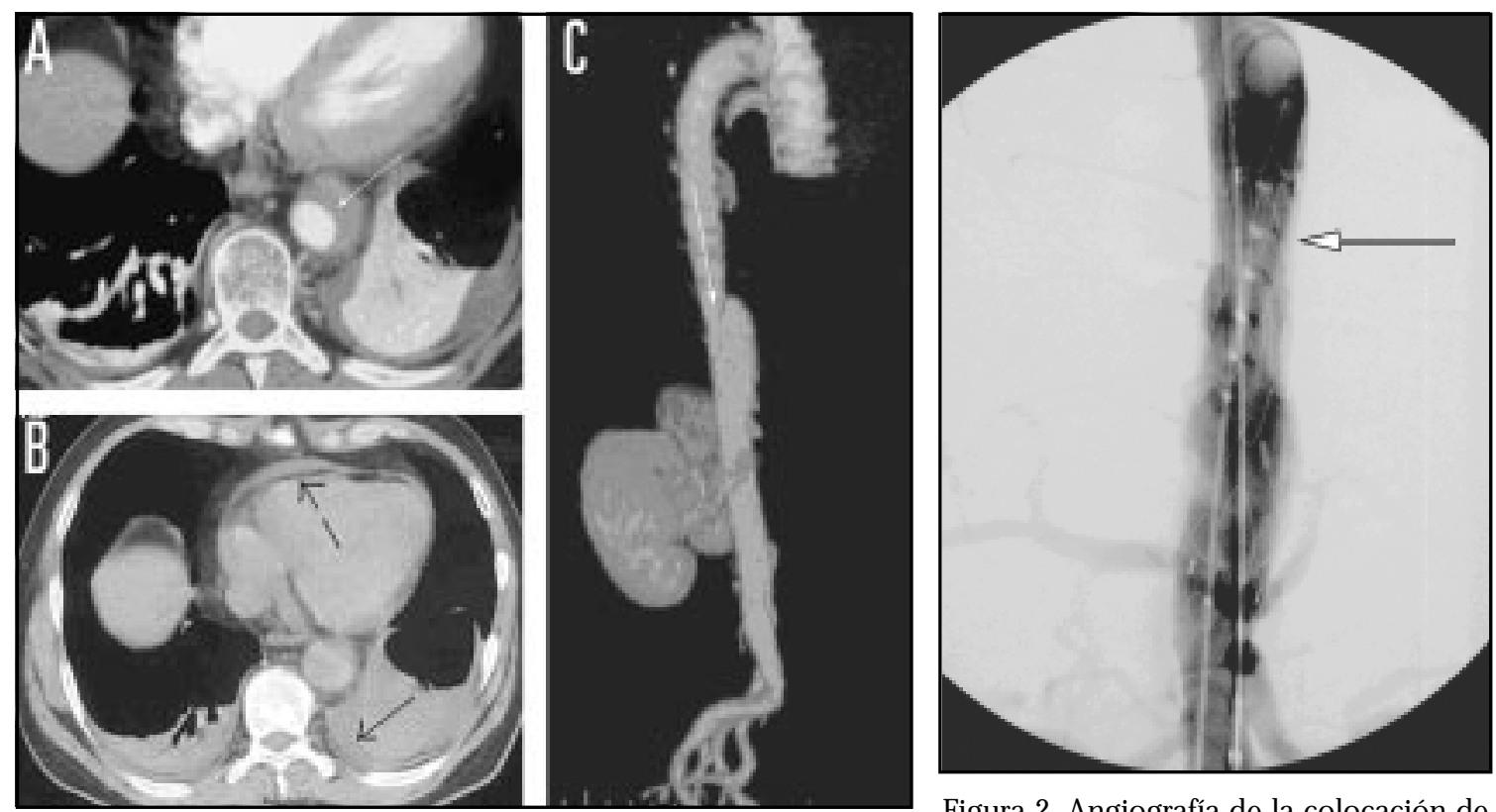

Figura 1. (a) Angio-Tac que demuestra disección de aspecto agudo de la aorta torácica descendente, con permeabilidad del falso lumen y flap de disección (flecha); (b) sin uso de contraste se demuestra hemopericardio (flecha discontinua) y leve hemotórax (flecha continua), lo que traduce complicación por rotura; (c) reconstrucción tridimensional de la aorta en toda su longitud, demostrando que el compromiso por el flap es sólo de aorta torácica descendente con extensión a segmento abdominal infrarrenal, por lo que constituye una disección tipo $\mathrm{B}$ complicada por rotura.

Figura 2. Angiografía de la colocación de la endoprótesis (flecha), la que demuestra una adecuada posición, con cobertura del sitio de fenestración proximal.

Figura 3. Control precoz, que demuestra (a) exclusión completa del falso lumen en aorta torácica, con cobertura adecuada del sitio de fenestración proximal; (b) un pequeño falso lumen residual infraabdominal, que no requiere tratamiento; (c) reconstrucción tridimensional en que se observa adecuada posición de la endoprótesis.

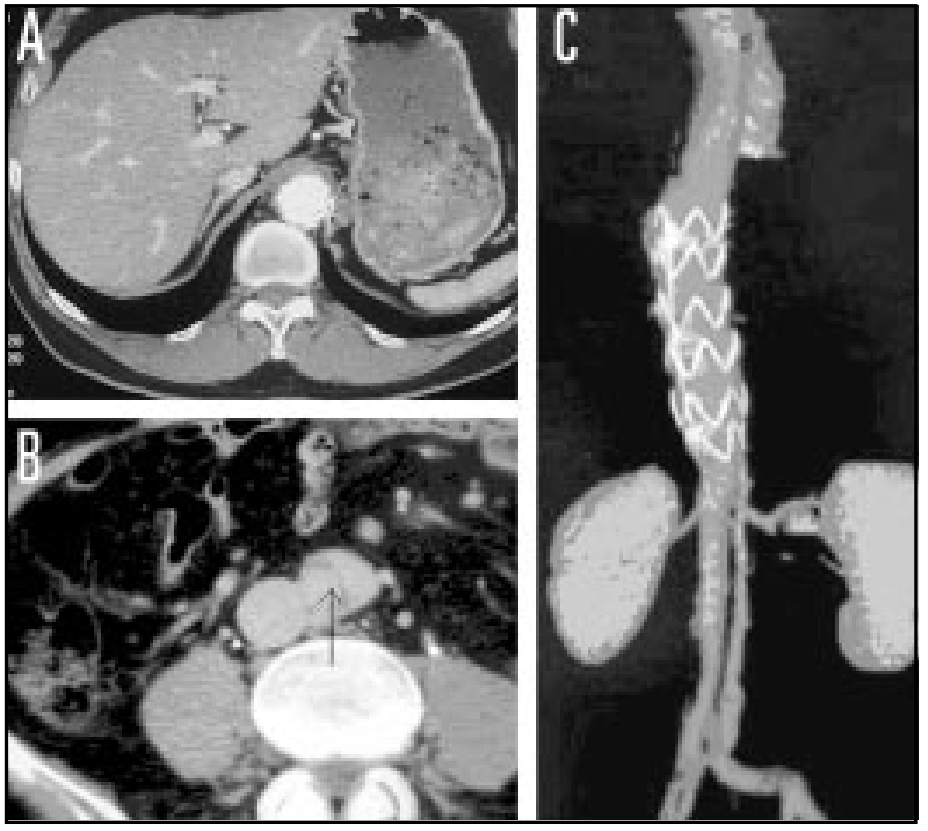

C A S O S

C L Ín I COS 


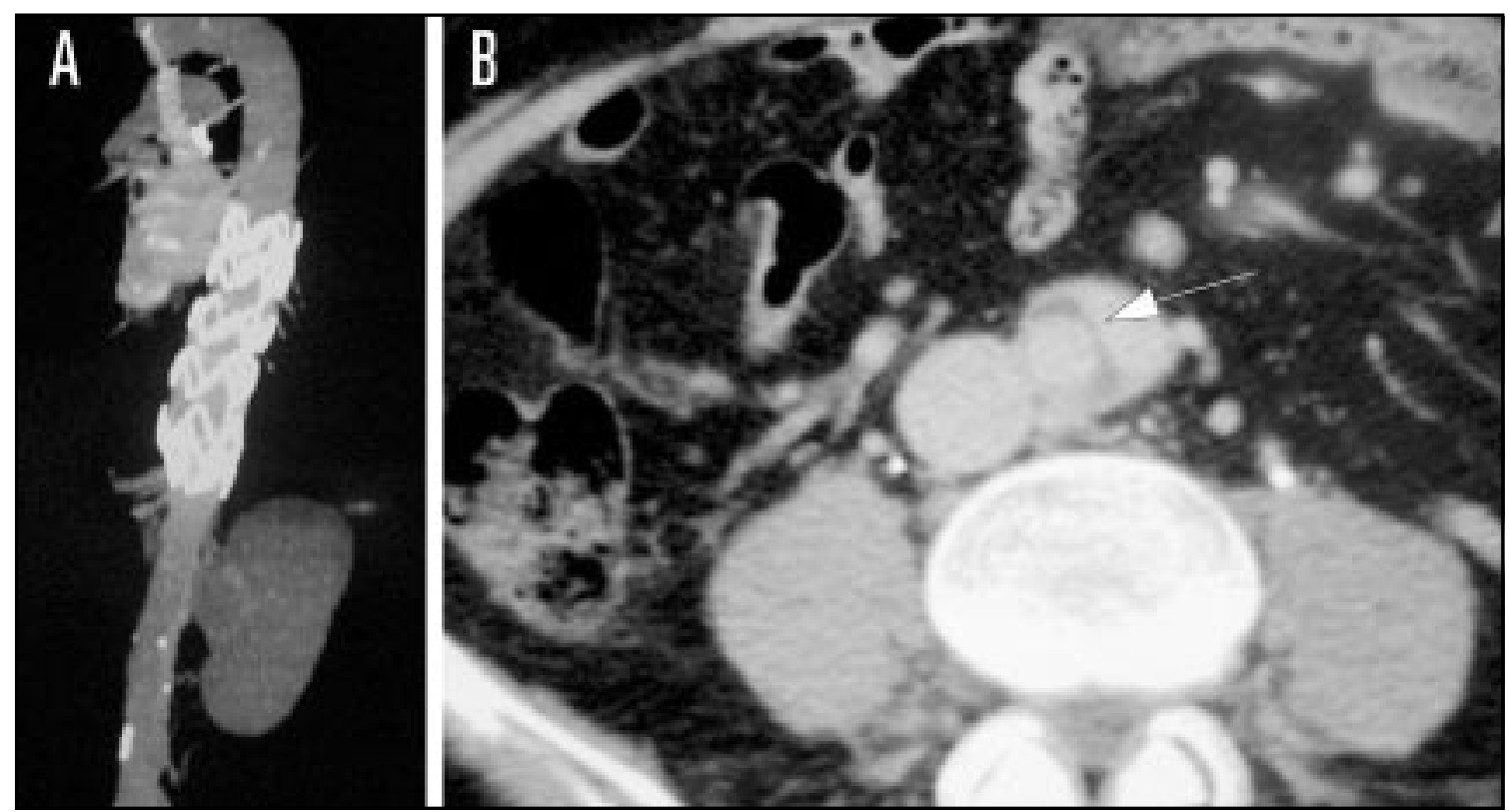

Figura 4. Control al año que presenta evolución favorable. (a) Reformateo que muestra adecuada ubicación de endoprótesis; (b) el pequeño lumen residual en aorta abdominal se encuentra estable con respecto a control precoz, sin requerir tratamiento específico.

tratamiento médico óptimo, mala perfusión con isquemia (visceral o medular) ${ }^{18}$.

En casos de disección aguda tipo B no complicada, la literatura sugiere el tratamiento conservador, sin embargo, la mortalidad a 30 días persiste alta, siendo de aproximadamente $20 \%$ con esta estrategia $^{19}$, con una probabilidad mayor a $30 \%$ de desarrollar aneurismas en relación con el falso lumen, en un período de 2-5 años ${ }^{20}$.

La reparación quirúrgica consiste en la colocación de prótesis en sitio de rotura, con mortalidad perioperatoria de, aproximadamente $40 \%$, en disección aguda tipo $\mathrm{B}^{21}$.

El uso de prótesis endovascular para el tratamiento de patología aórtica ha aparecido como técnica alternativa ${ }^{22,23}$. Recientemente, existen reportes acerca del uso de este procedimiento en pacientes con disección aguda de la aorta torácica descendente, con una mortalidad de $7 \%{ }^{24}$, bastante menor que la señalada para el tratamiento quirúrgico y sin isquemia medular, ya que no es necesario pinzar el cayado aórtico durante el procedimiento $^{18}$.
Los objetivos de la endoprótesis son: cubrir la fractura intimal proximal, trombosis del falso lumen y expansión del lumen verdadero ${ }^{15}$.

Debe existir una evaluación anatómica previo a la instalación de una endoprótesis, lográndose actualmente mediante el uso de Angio-Tac con multidetector. Se debe considerar la relación de la lesión con las ramas aórticas, y la condición de las arterias ilíacas y femorales, para el acceso vascular. El diámetro de las prótesis se calcula a partir de las imágenes obtenidas en la Angio-Tac. La imagen obtenida por Angio-Tac con multidector permite una certera medición del diámetro de las lesiones y las relaciones con las ramas aórticas, además de permitir evaluar las arterias ilíacas y femorales, que pueden ser las vías de acceso ${ }^{24}$. Se requiere que la prótesis tenga un diámetro 10-15\% mayor que el de la aorta, para lograr una buena aposición y evitar la migración.

La endoprótesis debe cubrir al menos $1,5 \mathrm{~cm}$ de aorta normal, tanto en el extremo distal como proximal, en el caso de la disección la prótesis puede ser más corta, dado que el objetivo es cubrir el sitio de fractura proximal ${ }^{24}$. 
La colocación se realiza a través de una pequeña arteriotomía de la arteria femoral común, por donde se ingresa el sistema introductorio, que se avanza en la aorta hasta la porción descendente de la aorta torácica. En caso de tener arterias femorales muy pequeñas (menores a $5 \mathrm{~mm}$ ), se puede realizar un abordaje retroperitoneal a la arteria ilíaca común.

El procedimiento se hace bajo control fluoroscópico. Para evitar la migración en el momento de la liberación, se disminuye la presión a menos de $90 \mathrm{mmHg}$ de valor sistólico ${ }^{23}$. Si la fractura intimal está a menos de $1,5 \mathrm{~cm}$ de la arteria subclavia izquierda, es posible cubrir el origen de esta arteria. Algunos autores recomiendan realizar un by-pass carótido-subclavio izquierdo, sin embargo, existen datos en la literatura, y en nuestra experiencia, que la cobertura de la arteria subclavia no causó síntomas, siendo necesario, la evaluación del flujo de la arteria vertebral contralateral previo al procedimiento 25 .

Las complicaciones precoces de la colocación de endoprótesis son: trauma aórtico o ilíaco, mala posición, falla renal, infarto al miocardio, accidente vascular encefálico y embolia arterial, ninguno de los cuales ha sido descrito en más de 7\%12,26.

\section{REFERENCIAS}

1. Chen K, Varon J, Wenker OC, Judge DK, Fromm RE JR, SteRNBACH GL. Acute thoracic aortic dissection: the basics. J Emerg Med 1997; 15: 859-67.

2. American Heart Association. Heart Disease and Stroke Statistical. 1998 Update. Dallas, TX.

3. ChirLLO F, Marchiori MC, ANdrIolo L. Outcome of 290 patients with aortic dissection: a 12 year multicentre experience. Eur Heart J 1990; 11: 3119.

4. Jamieson WR, Munro AI, Miyagishima RT, Auen P, Tyers GF, GereIN AN. Aortic disection: early diagnosis and surgical management are the keys to survival. Can J Surg 1982; 25: 145-9.

5. Hagan $P$, Nienaber $C$, Isselbacher E, Bruckman $D$, Karavite D, Russman P, Evangelista A. The International Registry of Acute Aortic Dissection (IRAD). JAMA 2000; 283: 897-903.
Uno de los problemas técnicos más importantes es la mala posición de la prótesis. Esto puede ocurrir por: a) la presión arterial alta durante la colocación o la dilatación con balón; b) el segmento en donde se coloca la prótesis es muy tortuoso o angulado ${ }^{24}$. El tratamiento del mal posicionamiento consiste en colocación de extensiones en caso de una cobertura incompleta de la lesión ${ }^{24}$.

El seguimiento a largo plazo de los pacientes se realiza con angio-Tac durante los primeros seis meses y luego anual ${ }^{24}$. De los elementos a considerar durante la evaluación, después de la colocación de la endoprótesis aórtica, están las filtraciones en relación a la prótesis (endoleak), que corresponde a la falla en excluir en forma efectiva el falso lumen.

En resumen, la colocación de prótesis endovasculares es un tratamiento seguro, efectivo, mínimamente invasivo y bien documentado para el tratamiento de patología de la aorta torácica descendente. Por tratarse de una técnica relativamente nueva, aún queda por resolver la durabilidad a largo plazo de las prótesis. Actualmente existen reportes en la literatura internacional que reflejan la ventaja del tratamiento endoluminal en casos de disección aórtica aguda complicada, en centros hospitalarios con médicos entrenados y experiencia en estas técnicas ${ }^{6,27,28}$.

6. JAMIESON WRE, JANUSZ MT, GudAs VM, BuRR LH, Fradet GJ, Henerson C. Traumatic rupture of the thoracic aorta: third decade of experience. Am J Surg 2002; 183: 571-5.

7. HochHeISER GM, Ciack DE, Morton JR. Operative technique, paraplegia and mortality after blunt traumatic aortic injury. Arch Surg 2002; 137: 434-8.

8. Jahromi AS, Kazemi K, Safar HA, Doobay B, Cina CS. Traumatic rupture of the thoracic aorta: cohort study and systematic review. J Vasc Surg 2001; 34: 1029-34.

9. Von Opell UO, Dunne TT, De Groot MK, Ziшa P. Traumatic aortic rupture: twenty year metanalysis of mortality and risk of paraplegia. Ann Thorac Surg 1994; 58: 585-93.

10. Jahromi AS, Kazemi K, Safar HA, Doobay B, Cina CS. Traumatic rupture of the thoracic aorta: cohort study and systematic review. J Vasc Surg 2001; 34: 1029-34. 
11. Fattori R, Napoli G, Lovato L, Grazia C, Piva T, Rocchi G. Descending Thoracic Aortic diseases: stent graft repair. Radiology 2003; 229: 176-83.

12. Dake MD, Miler DC, Semba CP, Mitchell RS, WALKER PJ, LidDELL RP. Transluminal placement of endovascular stent-grafts for the treatment of descending thoracic aortic aneurysms. N Engl J Med 1994; 331(suppl 26): 1729-34.

13. Matravers P, Morgan R, Bew A. The use of stent grafts for the treatment of aneurysm and dissections of the thoracic aorta: a single center experience. Eur J Vasc Endovasc Surg 2003; 26: 587-95.

14. CRiado FJ, Abul-Khoudoud. Endograft repair of acute aortic dissection. J Cardiovasc Surg 2005; 46: 107-12.

15. Alkadhi H, Wildermuth S, Desbioles L, Schertler T, Crook D, Marincek B, Boenm T. Vascular Emergencies of the Thorax after Blunt and Iatrogenic Trauma: Multi-Detector Row CT and Three-dimensional Imaging. RadioGraphics 2004; 24: 1239-55.

16. Chung JW, Park JH, Im JG, Chung MJ, Han MC, Ahn $\mathrm{H}$. Spiral CT angiography of the thoracic aorta. RadioGraphics 1996; 16: 811.

17. MILER DC. The continuing dilemma concerning medical versus surgical management of patients with acute type B dissection. Sem Thorac Cardiovasc Surg 1993; 5: 33-46.

18. Eiffteriades JA, Hartieroad J, Gusberg RJ, Salazar AM, BLACK HR, Kopf GS. Long-term experience with descending aortic dissection: the complication specific approach. Ann Thorac Surg 1992; 53: 11-21.

19. Kato M, Bai H, Sato K, Kawamoto S, Kaneko M, UEDA T. Determining surgical indications for acute Type B dissection based on enlargement of aortic diameter during the chronic phase. Circulation 1995; 92: (9 suppl) III 07-12.
20. Fann JI, Smith JA, Miuer DC, Mitchell RS, Moore KA, GRUNKEMEIER G ET AL. Surgical management of aortic dissection during a 30-year period. Circulation 1995; 92 (9 suppl): II113-II121.

21. Dake MD, Kato N, Mitchell RS, Semba CP, Razavi MK, Shimono T ET AL. Endovascular stent-graft placement for the treatment of acute aortic dissection. N Engl J Med 1999; 340: 1546-52.

22. Beregi JP, Prat A, Gaxotte V, Delomez M, McFadden EP. Endovascular treatment for dissection of the descending aorta. Lancet 2000; 356: 482-3.

23. Therasse E, Soulez G, Giroux MF, Perreault P, Bouchard L, Blair JF et al. Stent-Graft Placement for the Treatment of Thoracic Aortic Diseases RadioGraphics 2005; 25: 157-73.

24. Fattori R, Napol G, Lovato L, Grazia C, Piva T, RocCHI $G$ ET AL. Indications for, timing of, and results of catheter-based treatment of traumatic injury to the aorta. AJR Am J Roentgenol 2002; 179: 603-9.

25. Nienaber CA, Fattori R, Lund G, Dieckmann C, Wolf W, von Kodoltsch Y et aL. Nonsurgical reconstruction of thoracic aortic dissection by stent-graft placement. N Engl J Med 1999; 340: 1539-45.

26. Czermak BV, Waidengerger P, Peckermann R. Placement of endovascular stent-grafts for emergency treatment of acute disease of the descending thoracic aorta. AJR Am J Roentgenol 2002; 179: 337-45.

27. Amabile P, Collart F, Gariboldi V, Rolet G, Bartoli JM, Piquet P. Surgical versus endovascular treatment of traumatic thoracic aortic rupture. J Vasc Surg 2004; 40: 873-9.

28. Ott M, Charyk T, Lawlor K, Gray D, Forbes T. Management of blunt thoracic aortic injuries: Endovascular Stents versus Open Repair. J Trauma 2004; 56: 565-70. 